\title{
Resistance to topoisomerase cleavage complex induced lethality in Escherichia coli via titration of transcription regulators PurR and FNR
}

\author{
I-Fen Liu, Sandra Aedo and Yuk-Ching Tse-Dinh*
}

\begin{abstract}
Background: Accumulation of gyrase cleavage complex in Escherichia coli from the action of quinolone antibiotics induces an oxidative damage cell death pathway. The oxidative cell death pathway has also been shown to be involved in the lethality following accumulation of cleavage complex formed by bacterial topoisomerase I with mutations that result in defective DNA religation.
\end{abstract}

Methods: A high copy number plasmid clone spanning the upp-purMN region was isolated from screening of an E. coli genomic library and analyzed for conferring increased survival rates following accumulation of mutant topoisomerase I proteins as well as treatment with the gyrase inhibitor norfloxacin.

Results: Analysis of the intergenic region upstream of purM demonstrated a novel mechanism of resistance to the covalent protein-DNA cleavage complex through titration of the cellular transcription regulators FNR and PurR responsible for oxygen sensing and repression of purine nucleotide synthesis respectively. Addition of adenine to defined growth medium had similar protective effect for survival following accumulation of topoisomerase cleavage complex, suggesting that increase in purine level can protect against cell death.

Conclusions: Perturbation of the global regulator FNR and PurR functions as well as increase in purine nucleotide availability could affect the oxidative damage cell death pathway initiated by topoisomerase cleavage complex.

\section{Background}

DNA topoisomerases catalyze topological transformations of DNA by concerted breaking and rejoining of DNA strands via the formation of a covalent complex between the enzyme and cleaved DNA [1]. While the activities of topoisomerases are critical for vital cellular functions, topoisomerase enzymes are also vulnerable targets for cell killing because DNA rejoining by topoisomerases can often be inhibited by antibacterial or anticancer agents that are referred to as topoisomerase poisons $[2,3]$. Quinolones are widely used antibacterial drugs that lead to the accumulation of covalent cleavage complex formed by the bacterial type IIA topoisomerases, DNA gyrase and topoisomerase IV $[4,5]$. The accumulation of DNA gyrase covalent complex from the action of quinolones has been shown to induce an

\footnotetext{
* Correspondence: yuk-ching_tse-dinh@nymc.edu

* Correspondence: yuk-ching_tse-dinh@nymc.edu
Department of Biochemistry and Molecular Biology, New York Medical
} College, Valhalla, NY 10595, USA

(0) 2011 Liu et al; licensee BioMed Central Ltd. This is an Open Access article distributed under the terms of the Creative Commons Attribution License(http://creativecommons.org/licenses/by/2.0), which permits unrestricted use, distribution, and reproduction in any medium, provided the original work is properly cited. oxidative damage cell death pathway in E. coli as at least one of the potential mechanisms of cell killing [6-9]. The sequence of events following topoisomerase cleavage complex accumulation that leads to generation of reactive oxygen species remains unclear.

Although a specific poison for bacterial topoisomerase I remains to be identified, accumulation of topoisomerase I cleavage complex in $E$. coli has also been shown to lead to rapid cell death from the study of topoisomerase I mutants defective in DNA rejoining [10,11]. Similar to gyrase cleavage complex, topoisomerase I cleavage complex accumulation in E. coli induces the SOS response via the RecBCD pathway [12]. Increase in reactive oxygen species has been shown to also contribute to the cell death pathway initiated by accumulation of topoisomerase I cleavage complex [13]. Recombinant $E$. coli and Yersinia pestis topoisomerase I mutants that accumulate the covalent cleavage complex due to deficiency in DNA rejoining provide useful model systems for studying the physiological effect of topoisomerase- 
DNA cleavage complex accumulation. $Y$, pestis topoisomerase I (YpTOP1) is highly homologous to E. coli topoisomerase I, with the advantage of its dominant lethal recombinant clones being more stable in $E$. coli than comparable $E$. coli topoisomerase I mutant clones. The $Y$. pestis mutant topoisomerase I model system has been utilized to screen for $E$. coli genomic clones, that when present in high copy number on a plasmid, can confer resistance to topoisomerase cleavage complex induced cell killing. Additional experiments on an isolated clone demonstrated a novel mechanism of increased resistance to topoisomerase cleavage complex via titration of the transcription factors FNR and PurR by a high copy number plasmid clone of the intergenic region between $u p p$ and purM. This plasmid clone also increased bacterial resistance to norfloxacin that induces the accumulation of the type IIA topoisomerase covalent cleavage complex. FNR regulates transition between anaerobic and aerobic conditions [14,15]. Genome-wide expression analysis has previously shown that FNR contributes to the repression of a number of genes induced by oxidative stress conditions [16,17]. PurR is a suppressor of purine biosynthesis. Titration of the FNR and PurR transcription factors by the high copy number clone is expected to increase the expression level of genes normally suppressed by these two regulators. These results provide further insights into the oxidative cell death pathways initiated by topoisomerase cleavage complex accumulation.

\section{Results}

Isolation of clone pAQ5 containing the upp-purMN region in selection for resistance to topoisomerase I cleavage complex mediated cell death

After transformation of E. coli strain BW117N with the $E$. coli genomic DNA library generated with the pCRXL-TOPO cloning system, four different plasmid clones isolated from colonies obtained on LB plates with $0.002 \%$ arabinose were confirmed to increase resistance to the dominant lethal effect of the mutant $Y$. pestis topoisomerase I, YpTOP1-D117N [10]. Detailed analysis of the clone pAQ5 containing the upp-purMN region of E. coli chromosome (corresponding to nucleotides 2618398-2620765 of E. coli MG1655 sequence, Figure 1a) is described here. Strain BW $117 \mathrm{~N}$ is under strong selective pressure to eliminate expression of the dominant lethal mutant YpTOP1-D117N. Subsequent analysis of the effect of clone pAQ5 or its derivatives was therefore carried out with strain BW27784 carrying plasmid pAYTOP128 expressing YpTOP1 with the less lethal G122S mutation that also leads to accumulation of the topoisomerase I cleavage complex [11]. Clone pAQ5 was found to increase survival following arabinose induction of this mutant YpTOP1 by 63 -fold compared to the control empty vector (Table 1 ). This clone (Figure 1a) contains the entire purM (5'-phosphoribosyl-5aminoimidazole synthetase) coding sequence (26192192620256), part of the purN (phosphoribosylglycinamide formyltransferase) coding sequence (2620256-2620894), and part of the upp (uracil phosphoribosyltransferase) coding sequence (2618268-2618894), plus the intergenic regulatory region between the $u p p$ and $p u r M N$ genes (2618946-2619178). Analysis of total cellular protein by western blot showed that clone pAQ5 did not confer resistance by decreasing the expression level of mutant topoisomerase I after arabinose induction (Figure 2a).

\section{Analysis of resistance to topoisomerase I cleavage complex conferred by upp-purMN intergentic region}

To determine the basis of resistance from clone pAQ5, derivatives of pAQ5 were constructed by cloning of specific PCR amplified DNA into pCR-XL-TOPO vector. These include clones $\mathrm{PAQ} 5-1$ with purM and the intergenic region, pAQ5-2 with uppA and the intergenic region, and pInter, with the intergenic region alone (Figure 1a). These clones were transformed into strain BW27784 containing pAYTOP128 expressing mutant $Y$. pestis topoisomerase I deficient in DNA religation due to the TOPRIM G122S mutation to investigate the effect of the clones on viability following induction of mutant topoisomerase I. The results (Table 1) showed that the intergenic region alone in clone pInter was sufficient to confer resistance to the mutant topoisomerase I. Western blot analysis confirmed that the protective effect of pInter was also not due to reduction in expression level of mutant topoisomerase I (Figure 2b).

Examination of this intergenic sequence showed that it includes the binding site sequences of two transcription factors, FNR and PurR (Figure 1b). The FNR binding sequence, TTGACTTTAGTCAA versus the TTGAT$\mathrm{N}_{4} \mathrm{ATCAA}$ consensus sequence [18-20], is located 61.5 nucleotides upstream of the upp transcription start site. The PurR binding sequence, CGCAAACGTTTGCTT, versus the consensus PurR operator sequence of CGCAAACGTTTNCNT [21], is located 28 nucleotides upstream of the purM gene. FNR acts as a dual transcription regulator that activates certain genes required for anaerobic growth and represses many genes required for aerobic growth [22]. Its interaction with the upppurMN region has been reported previously [19]. PurR negatively regulates the transcription of genes involved in purine and pyrimidine nucleotide synthesis including purMN $[21,23,24]$. We therefore hypothesize that the high copy number pInter could titrate these transcription factors to relieve the repression of other $E$. coli genes encoded on the chromosome. To test this hypothesis, these binding sites were eliminated individually by 
A

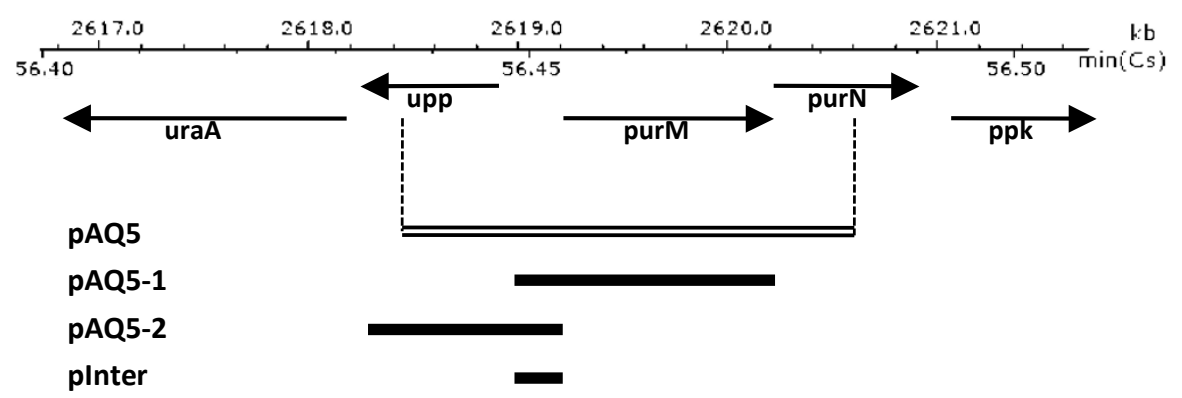

B

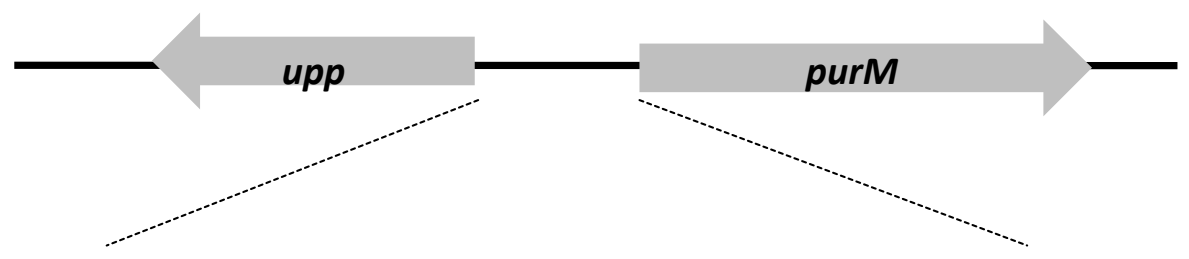

ACTCTTTCTCCTTTGAGGGGCAGCCACAAAAAAAATCGACGGATTAT

FNR

ACCTCCTTTCTTCAAGGCGGCAATATTCTTTTCG ITGACTTTAGTCAA

AATGATAACGGTTTGAGATAAAGTTATTTTATATTCAGATGGTTATG

AAAGAAGATTATTCCATCCGAAAACTAACCTTTACCCTGGCACAAGT

CTTCTTTCGCCGCGCGCCTGGGGAAAAGACGTGCAAAAAGGTTGTGT

PurR

AAAGCAGTCT GGCAAACGTTTGCTTCCCTGTTAGAATTGCGCCGAA

TTTTATTTTTCTACCGCAAGTAACGCGTGGGGACCCAAGCA

C Deletion of FNR binding site in plnterD1

5'-CGCAACGTTTGCTTT-

Deletion of PurR binding site in plnterD2

5'

Figure $1 \mathrm{E}$. coli chromosomal DNA insert in high copy plasmid clone pAQ5 and its derivatives (a) Clone pAQ5 containing sequence in the upp-purM-purN region was selected from an E. coli genomic DNA plasmid library for resistance to cell killing mediated by mutant topoisomerase I YpTOP1-D117N expressed in BW117N. PCR was used to amplify the intergenic sequence shown in (b) for cloning into pCRTOPO-XL cloning vector in the construction of plnter. The sequence of the FNR and PurR binding site deleted in plnterD1 and plnterD2 is shown in (c). 
Table 1 Effect of high copy plasmid clones on survival following accumulation of mutant topoisomerase I cleavage complex

\begin{tabular}{ll}
\hline Plasmid & Survival Ratio \\
\hline pCRII vector & $7.85 \times 10^{-5} \pm 1.19 \times 10^{-5}$ \\
\hline pAQ5 & $4.95 \times 10^{-3} \pm 1.55 \times 10^{-3}$ \\
\hline pAQ5-1 & $4.92 \times 10^{-3} \pm 1.20 \times 10^{-3}$ \\
\hline pAQ5-2 & $1.25 \times 10^{-2} \pm 2.48 \times 10^{-3}$ \\
\hline plnter & $1.90 \times 10^{-2} \pm 4.12 \times 10^{-3}$ \\
\hline plnterD1 & $4.22 \times 10^{-3} \pm 1.02 \times 10^{-3}$ \\
\hline plnterD2 & $5.19 \times 10^{-4} \pm 1.73 \times 10^{-4}$
\end{tabular}

E. coli BW27784 carrying PAYTOP128 was transformed with high copy number plasmid shown in the table. Cultures were grown to exponential phase with shaking, then treated with $0.002 \%$ arabinose for $2.5 \mathrm{~h}$ before serial dilution and plating on LB plates with antibiotics and $2 \%$ glucose

Survival ratio was determined by calculating the ratio of the viable colony counts obtained from the induced cultures versus the viable counts from noninduced culture. The results represent the average and standard errors from at least three experiments

site-directed mutagenesis (Figure 1c). Nucleotides TGACTTTAGTCA were deleted from the FNR binding site to result in plasmid pInterD1. Nucleotides AAACGTTTGCTT were deleted from the PurR binding site to result in plasmid pInterD2. Measurement of cell viability following induction of mutant topoisomerase from pAYTOP128 showed that elimination of either of these two binding sites reduced the protective effect of pInter, (Table 1). Comparison of the growth curves of these strains (Figure 2c) showed that while cells transformed with pInter and pInterD1 grew to a lower density at saturation, the initial growth rates of these strains are similar. The slightly slower growth rate of cells transformed with pInterD1 was not statistically significant and since pInterD1 conferred a lesser degree of resistance than pInter, the difference in viability following accumulation of topoisomerase I cleavage complex cannot be accounted for simply as due to growth inhibition.

Effect of high copy number plasmid clone pInter on sensitivity to norfloxacin BW27784 transformed with the high copy number plasmid clones pAQ5 or pInter were treated with the gyrase inhibitor norfloxacin to determine if the plasmids could confer resistance also to cell death mediated by type II topoisomerase cleavage complex. The results (Table 2) showed that these plasmids could confer $\sim 30$-fold higher survival rates than the control vector. Deletion of either the FNR or PurR binding site from pInter decreased the protective effect, demonstrating that titration of these transcription factors could result in increased resistance to norfloxacin. It should be noted that pInterD1 conferred more protection than pInterD2 to mutant topoisomerase I killing (Table 1) and the opposite was true for norfloxacin killing (Table 2).

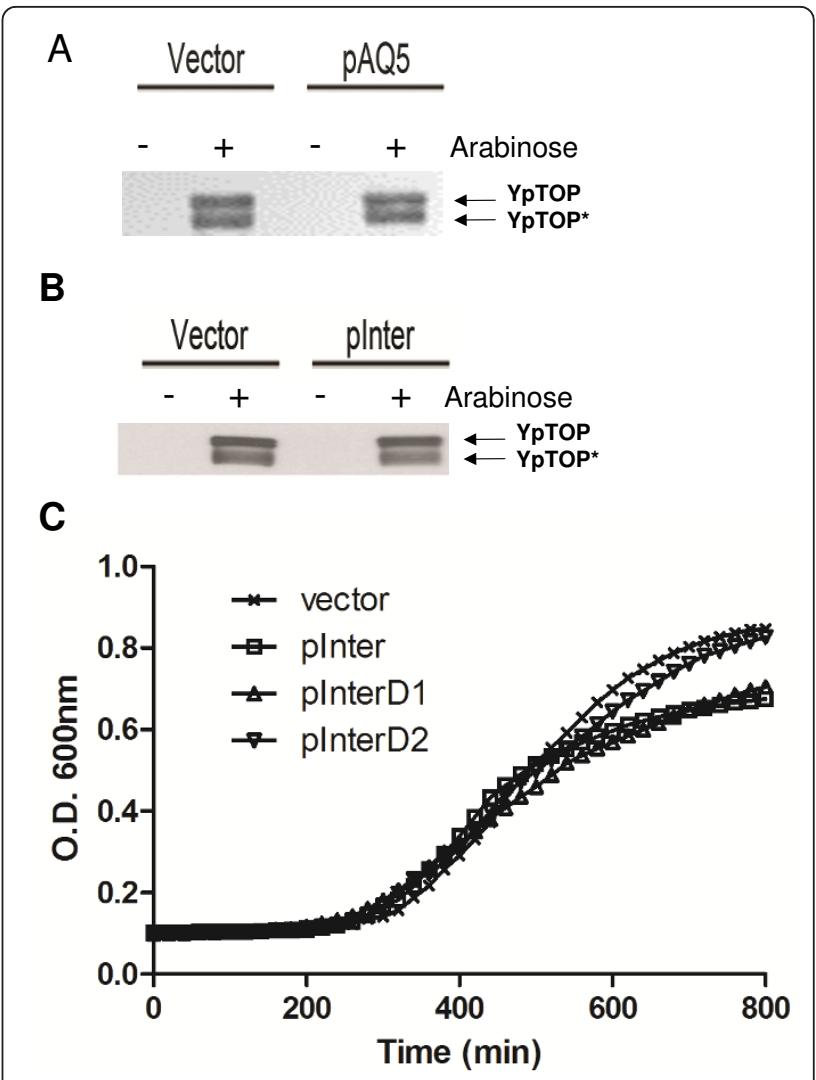

Figure 2 Effect of plasmid clones on recombinant mutant $Y$. pestis topoisomerase I expression and growth rates Western blot analysis of expression of mutant $Y$. pestis topoisomerase I in the presence of control vector and clone PAQ5 (a) or plnter (b). Exponential phase cultures were treated with $0.002 \%$ arabinose for $2.5 \mathrm{~h}$. Total cellular protein was analyzed by SDS PAGE and Western blot with mouse monoclonal antibodies against $E$. coli topoisomerase I (ECTOP). This antibody recognizes the highly homologous Y. pestis topoisomerase I (YpTOP) and its partially degraded product (YpTOP*).(C) Growth of BW27784 transformed with vector, plnter, plnterD1 and plnterD2 in LB. Absorbance was measured in a 96 well microplate at $37^{\circ} \mathrm{C}$ every 20 min using the Perkin Elmer 7000 Plus BioAssay Reader with the filter set at $590 \mathrm{~nm}$ and shaking for $10 \mathrm{~min}$ before each measurement.

Table 2 Effect of high copy plasmid clones on survival following treatment with norfloxacin

\begin{tabular}{ll}
\hline Plasmid & Survival Ratio \\
\hline pCRII vector & $2.14 \times 10^{-5} \pm 4.1 \times 10^{-6}$ \\
\hline pAQ5 & $7.57 \times 10^{-4} \pm 2.14 \times 10^{-4}$ \\
\hline plnter & $6.12 \times 10^{-4} \pm 1.28 \times 10^{-4}$ \\
\hline plnterD1 & $8.41 \times 10^{-5} \pm 3.55 \times 10^{-5}$ \\
\hline plnterD2 & $1.11 \times 10^{-4} \pm 2.01 \times 10^{-5}$ \\
\hline
\end{tabular}

E. coli BW27784 transformed with high copy number plasmid was grown to exponential phase with shaking. Cultures were treated with $250 \mathrm{ng} / \mathrm{ml}$ norfloxacin for $2 \mathrm{~h}$ before serial dilution and plating on LB plates with kanamycin

Survival ratio was determined by calculating the ratio of the viable colony counts obtained from the treated cultures versus the viable counts from untreated culture. The results represent the average and standard errors from at least three experiments 
Protective effect from adenine addition

The protective effect from titration of PurR could be due to increased availability of purine nucleotides. This was tested by growth of BW27784 transformed with pAYTOP128 in minimal media. Greater than 3 logs of loss of viability could be measured at $2 \mathrm{~h}$ after induction of mutant topoisomerase I expression by $0.0002 \%$ arabinose (Figure 3a). The presence of $100 \mu \mathrm{g} / \mathrm{ml}$ adenine in the growth medium increased the number of viable colonies by 30 -fold at $2 \mathrm{~h}$ after arabinose addition. The presence of adenine did not affect expression level of mutant topoisomerase I as determined by western blot (Figure 3B).

To determine if addition of adenine affects sensitivity to norfloxacin, BW27784 cells grown in minimal medium with different adenine concentrations were first evaluated by examining growth inhibition by norfloxacin. Increased resistance to growth inhibition by norfloxacin was observed in the presence of $250 \mu \mathrm{g} / \mathrm{ml}$

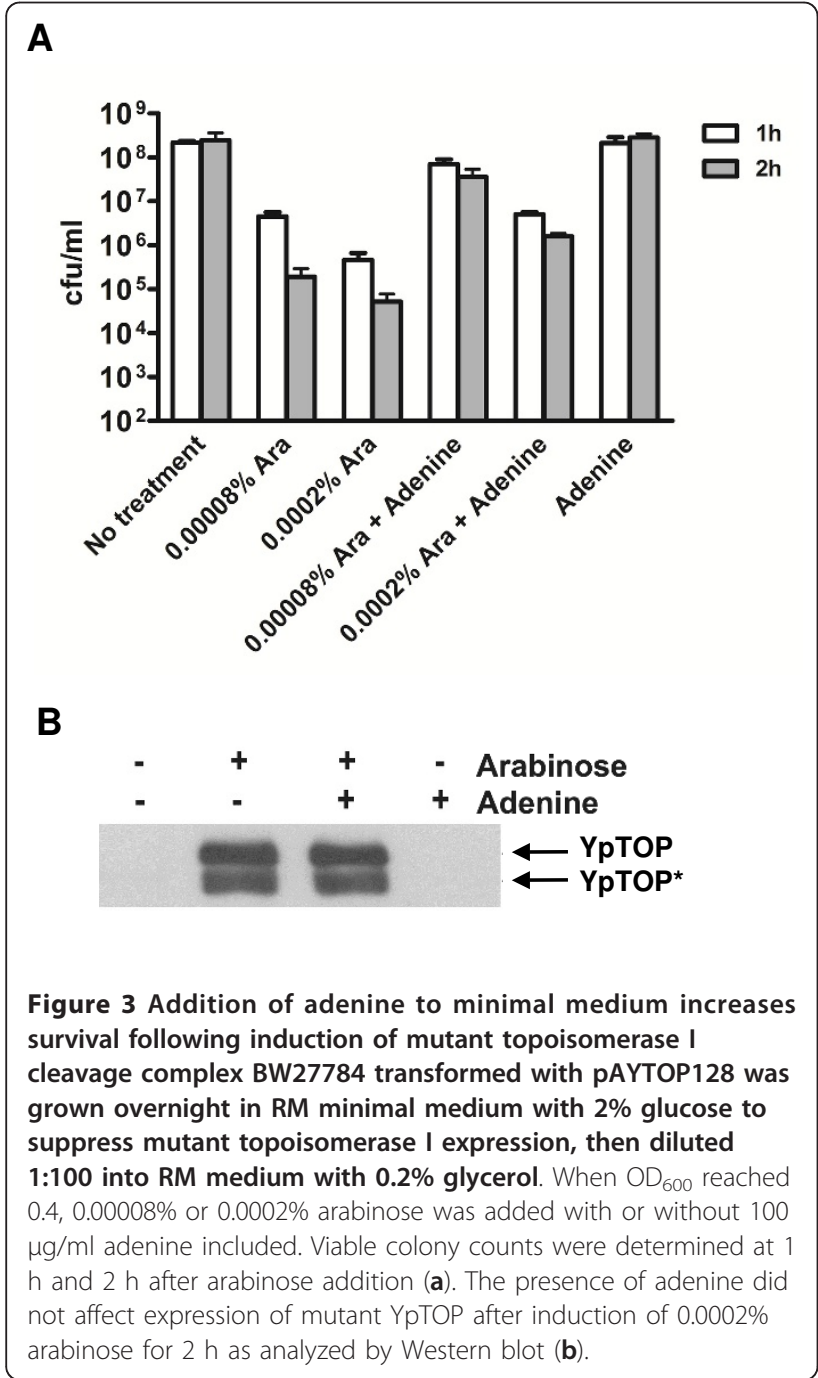

adenine (Figure 4a). Growth of BW27784 in the absence of norfloxacin was not affected significantly by the presence of adenine. Viable colony counts at $3 \mathrm{~h}$ after norfloxacin treatment were then measured and found to be increased 24-fold by the presence of adenine (Figure 4b).

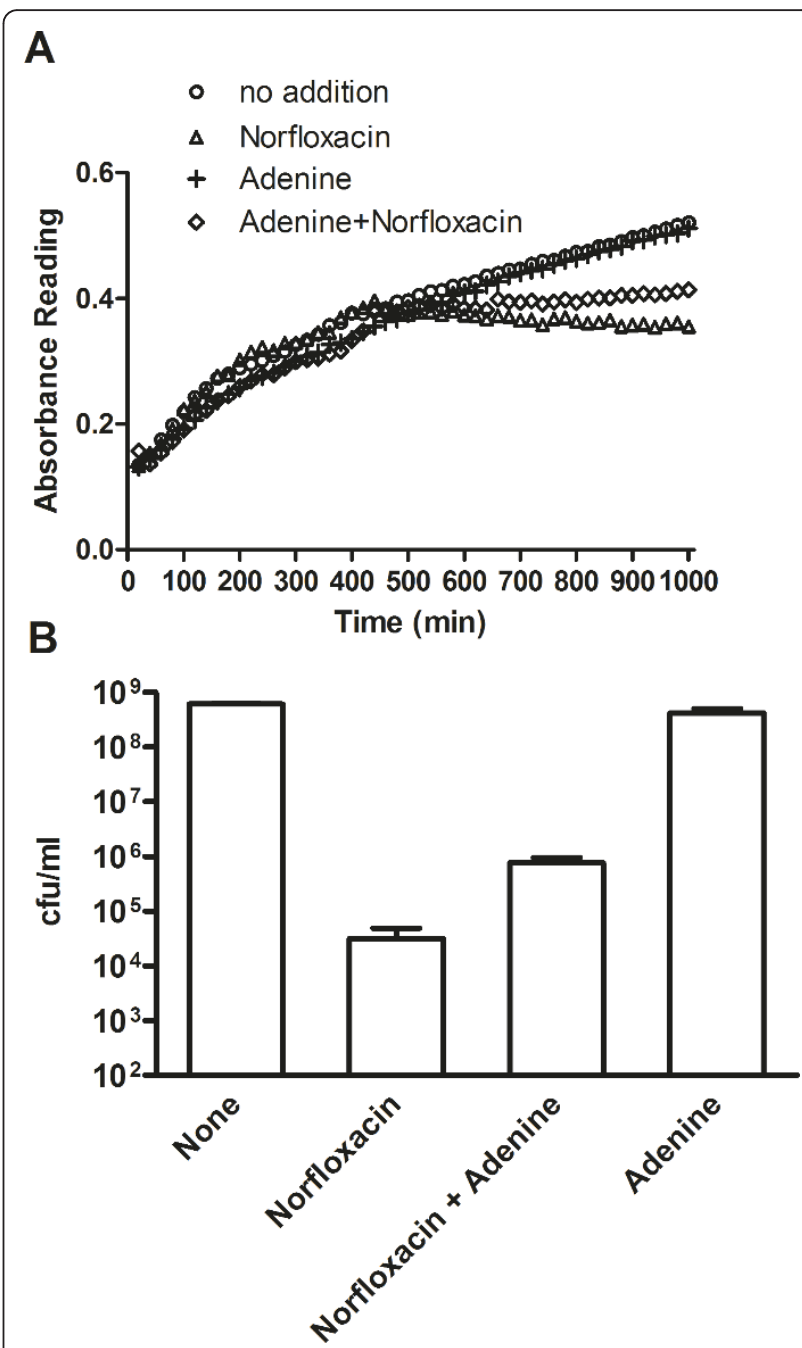

Figure 4 Decreased sensitivity to norfloxacin from addition of adenine BW27784 was grown overnight in RM minimal medium with $0.2 \%$ glycerol and then diluted 1:100 and grown to exponential phase. (a) The exponential phase culture was diluted twofold with RM medium with (o) no addition, (+) $250 \mu \mathrm{g} /$ $\mathrm{ml}$ adenine, $(\Delta) 120 \mathrm{ng} / \mathrm{ml}$ norfloxacin, or $(\Delta) 120 \mathrm{ng} / \mathrm{ml}$ norfloxacin and $250 \mu \mathrm{g} / \mathrm{ml}$ adenine. Absorbance was measured at $37^{\circ} \mathrm{C}$ every 20 min using the Perkin Elmer 7000 Plus BioAssay Reader with the filter set at $590 \mathrm{~nm}$ and shaking for 10 min before each

measurement. (b) Exponential phase culture was treated with 200 $\mathrm{ng} / \mathrm{ml}$ norfloxacin with or without $250 \mu \mathrm{g} / \mathrm{ml}$ adenine along with controls with no treatment or adenine alone. After $3 \mathrm{~h}$ at $37^{\circ} \mathrm{C}$, viable colony counts were determined by dilution and plating on LB plates. 
The high copy number intergenic region clone decreases the level of hydroxyl radicals following norfloxacin treatment

The high copy number pInter resulted in $\sim 30$-fold higher ratio of viability after treatment with norfloxacin when compared to control plasmid with no insert (Table 2). Bactericidal antibiotics have been shown to initiate formation of reactive oxygen species in their cell killing mechanism $[7,8,25]$, and hydroxyl radicals formation has been shown to be involved in bacterial cell death following topoisomerase I cleavage accumulation [13]. We hypothesize that the high copy number of the upp-purMN intergenic region modulates cellular metabolism to reduce the formation of reactive oxygen species upon accumulation of topoisomerase I cleavage complex. Formation of hydroxyl radicals was followed by increase in fluorescence intensity from reporter HPF [7]. The results (Figure 5) showed that at $2 \mathrm{~h}$ after addition of $250 \mathrm{ng} / \mathrm{ml}$ of norfloxacin, HPF fluorescence intensity from hydroxyl radicals in BW27784 cells transformed with pInter was reduced compared to HPF fluorescence from BW27784 transformed with vector after drug treatment.

Effect of chromosomal fnr and purR mutations on sensitivity to topoisomerase I cleavage complex accumulation

To support the hypothesis that the protective effect from pInter is due to the titration of the transcription factors FNR and PurR, chromosomal mutations eliminating the activity of the fur and purR genes were introduced into BW27784 by $\mathrm{P} 1$ transduction resulting in strains IFL6 $(\Delta f n r)$ and IFL7 ( $\Delta p u r R)$. Viable colony

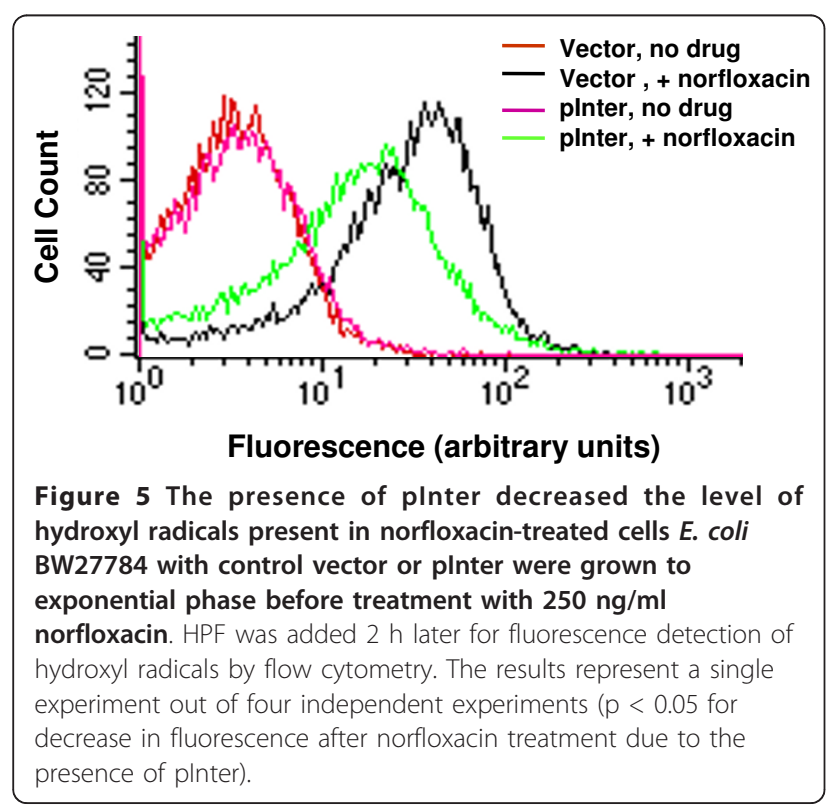

counts were measured following induction of mutant topoisomerase I expression from pAYTOP128. The results showed that in agreement with the hypothesis, higher rates of survivals were observed in the absence of FNR or PurR activity, with a greater effect from the purR mutation (Figure 6a). Western blot analysis was used to confirm that the mutations did not affect induction of mutant topoisomerase I expression (Figure 6b). It is consistent that PurR loss, either by purR mutation shown in Figure 6a or titration by high copy of its binding site in pInterD1 and other plasmids shown in Table 1 , increases resistance.

The protective effect of $\Delta f n r$ mutation was greater under low oxygen conditions

The genes suppressed by FNR directly or indirectly via the sRNA FnrS [26,27] include many aerobic metabolic genes as well as genes involved in removal of reactive oxygen species such as katE, $\operatorname{sod} A$ and $\operatorname{sod} B$ [16]. Hydroxyl radicals generated from superoxide have been shown to be involved in the cell killing pathway initiated

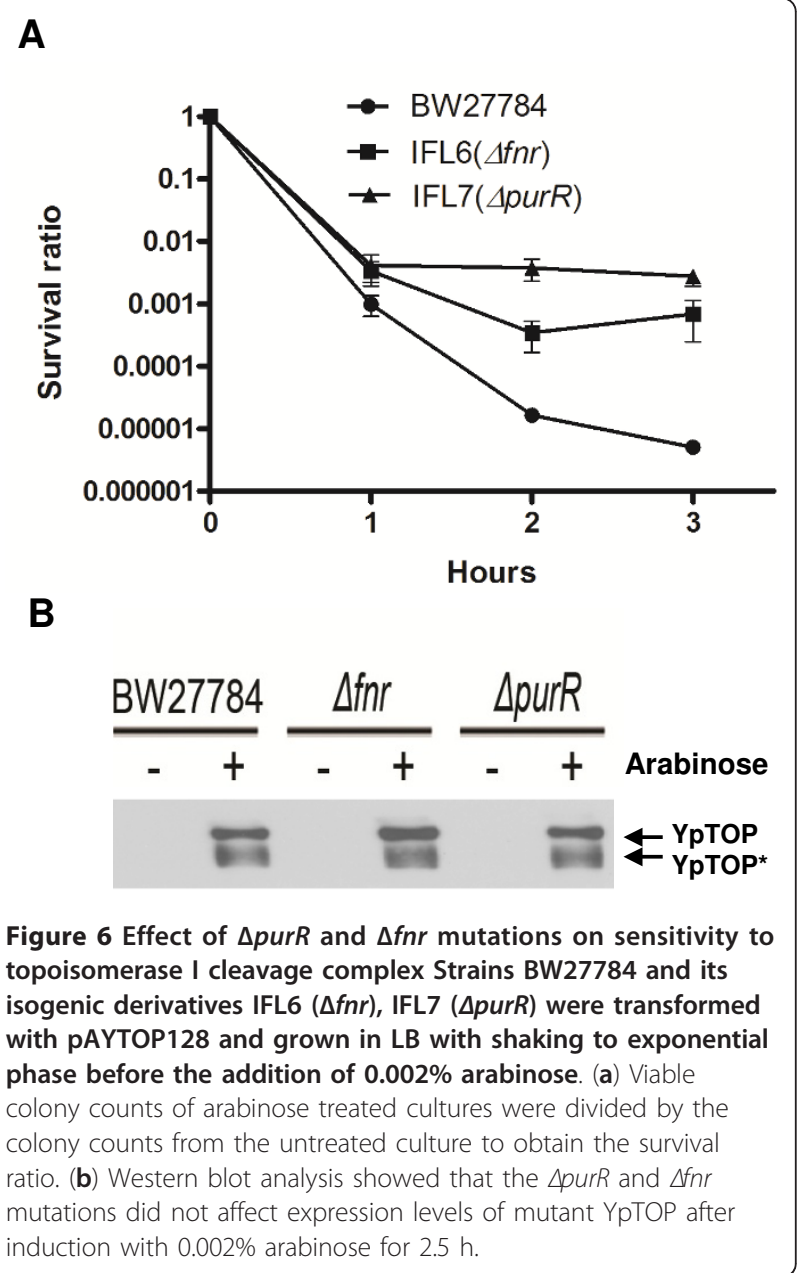


by topoisomerase I cleavage complex [13]. Cells in liquid cultures have been incubated with shaking at $215 \mathrm{rpm}$ in our experiments carried out so far. Gene regulation by FNR is responsive to low level of oxygen [22,28]. We therefore modified our experimental conditions to decrease oxygen availability. BW27784 or IFL6 $(\Delta f n r)$ cells were grown without shaking in a closed vessel until $\mathrm{OD}_{600}=0.4$. After addition of arabinose to induce mutant topoisomerase I expressed by pAYTOP128, the culture was divided into two portions and incubation was continued with and without shaking. Measurement of survival ratio (ratio of viable colonies compared to control culture with no arabinose added) shown in Table 3 indicated that for BW27784, the survival ratio after induction of mutant topoisomerase I is higher in culture without shaking (around tenfold), likely due to lower level of reactive oxygen species. The protective effect from the $\Delta f n r$ mutation was more prominent when oxygen was limiting versus when oxygen was available. This is in agreement with the active role of FNR in gene regulation under anaerobic conditions.

However, chromosomal $\Delta p u r R$ and $\Delta f n r$ mutations were found to have little effect on the viable colony counts at 1 and $2 \mathrm{~h}$ after treatment with up to $250 \mathrm{ng} /$ $\mathrm{ml}$ norfloxacin (data not shown). Greater than 1000-fold lower bactericidal rates were observed for BW27784 with oxygen limitation when compared to incubation with oxygen after treatment with norfloxacin, in agreement with previous report of decreased norfloxacin sensitivity under anaerobic conditions [29]. It is therefore not feasible to investigate any potential protective effect from pInter or the $\Delta f n r$ mutation under low oxygen conditions.

\section{Discussion}

A segment of E. coli chromosomal DNA spanning the upp-purMN region was selected from a high copy number plasmid library of $E$. coli genomic DNA fragments based on its ability to confer resistance to cell killing

Table 3 Protective effect of $\Delta f n r$ mutation for cell killing initiated by mutant topoisomerase I cleavage complex accumulation under aerobic and low oxygen conditions

\begin{tabular}{lll}
\hline \multicolumn{2}{l}{ Survival Ratio } & \\
\hline & Aerobic & Low Oxygen \\
\hline BW27784 & $1.18 \times 10^{-4} \pm 7.7 \times 10^{-5}$ & $1.07 \times 10^{-3} \pm 4.7 \times 10^{-4}$ \\
\hline IFL6 & $1.30 \times 10^{-3} \pm 3.1 \times 10^{-4}$ & $8.15 \times 10^{-2} \pm 3.1 \times 10^{-3}$ \\
\hline$\Delta$ fnr effect & 11 -fold & 76 -fold \\
\hline
\end{tabular}

Cultures were grown to exponential phase without shaking, then treated with $0.002 \%$ arabinose for $2.5 \mathrm{~h}$ under either aerobic or low oxygen conditions before serial dilution and plating on LB plates with antibiotics and $2 \%$ glucose. Survival ratio was determined by calculating the ratio of the viable colony counts obtained from the induced cultures versus the viable counts from non-induced culture. The results represent the average and standard errors from at least three experiments mediated by accumulation of topoisomerase I cleavage complex. The intergenic region of upp-purMN was found to protect against bacterial cell death initiated by both type I and type II covalent topoisomerase-DNA cleavage complex. Deletion of the binding sites for FNR and PurR decreased the protective effect, suggesting that the protective effect we observed for pInter resulted from titration of the transcription regulators FNR and PurR.

PurR is a repressor of purine biosynthesis in E. coli [19]. The hypothesis that the protective effects observed from the high copy number plasmid pInter is related to purine nucleotide pool availability is supported by the increased viability when adenine was added to defined medium. The $\triangle p u r R$ mutation resulted in up to $475-$ fold higher survival rate following topoisomerase I covalent cleavage complex accumulation. Although pInter could increase survival rate following norfloxacin treatment, the $\triangle p u r R$ chromosomal mutation did not affect norfloxacin sensitivity. Deletion mutation of a global transcription regulator is likely to affect the many metabolic genes under its regulation differently than titration of the global transcription regulator by the presence of its binding site on a high copy number plasmid. Chromosomal PurR recognition sites with the strongest binding affinity for PurR might still be repressed by PurR even in the presence of pInter but they would be depressed in the $\triangle p u r R$ background. The cell death pathways initiated by type IA and type IIA topoisomerases may be affected to different degrees by the change in metabolic gene expression resulting from $\triangle p u r R$ mutation. The level of cellular ATP and $\mathrm{NAD}^{+} / \mathrm{NADH}$ ratio are factors that could influence the induction of the reactive oxygen species following accumulation of the topoisomerase cleavage complex $[7,8]$.

FNR is a global regulator for the response of many genes to oxygen level $[22,28]$. It can activate or repress different genes directly by binding to the upstream regulatory region [19]. FNR also activates the transcription of the small non-coding RNA FnrS which negatively regulates the expression of multiple genes, including many that encode enzymes with functions linked to oxidative stress [26,27]. The presence of its binding site on pInter was responsible for part of the resistance to topoisomerase I cleavage complex mediated cell killing conferred by this high copy number plasmid. The oxygen level in the culture decreased as cell growth approached stationary phase even with shaking, probably resulting in partial activity of the FNR protein. Regulatory effect of FNR on transcription of acetyl coenzyme A synthetase gene in E. coli has been previously observed under conditions that are not strictly anaerobic [30]. We showed that the protective effect of the $\Delta f n r$ mutation on cell death following topoisomerase I 
cleavage complex accumulation was more prominent under low oxygen condition, consistent with the increased activity of FNR expected when oxygen is limiting. FNR may influence cell death pathway initiated by topoisomerase cleavage complex by suppressing the genes that can enhance the response to reactive oxygen species implicated in the cell death pathway. Alternatively, decrease in FNR activity may alter the metabolic state of the cell, so that it is less susceptible to the oxidative damage cell death pathway.

In future studies, it would be informative to express FNR and/or PurR in the corresponding deletion mutants under the control of an inducible promoter. This would allow examination of promoter occupation across the genome and correlate global gene expression pattern with sensitivity to the oxidative damage cell death pathway.

\section{Methods}

\section{Bacterial strains and plasmids}

Genomic DNA E. coli strain YT103 was used to generate the chromosomal fragment library. It has $y d e A:: k a n$ and $\Delta a r a$ mutations to avoid having clones in the library that are known to decrease expression from the arabinose inducible BAD promoter [31]. Sensitivity to topoisomerase I cleavage complex mediated cell death was measured in E. coli strain BW27784 and its derivatives. This genetic background allows uniform expression of recombinant mutant topoisomerase I under the control of the BAD promoter in response to arabinose [32]. The YpTOP1-D117N clone with the highly lethal Asp to Asn mutation at the first aspartate of the TOPRIM DxDxxG motif [33] was integrated into the chromosome in strain BW117N [10]. Mutant YpTOP1 with the Gly to Ser mutation at position G122S of the TOPRIM motif was expressed from plasmid pAYTOP128 [11]. Other chromosomal mutations were introduced into E. coli BW27784 by P1 transduction. PCR amplification of specific E. coli chromosomal fragments for cloning, and site-directed mutagenesis of the plasmid encoded sequence, were carried out using $P f u$ II Ultra DNA polymerase (from Stratagene). All strains and plasmids used in this study are listed in Table 4. LB medium was used for culture unless otherwise stated.

\section{Screening of clones conferring resistance to topoisomerase I cleavage complex}

E. coli YT103 chromosomal fragments, with sizes between 2.5 and $4.5 \mathrm{kbp}$, generated from partial Sau3A1 digestion and sonication were gel purified and used to generate a high copy number plasmid library with the pCR-XL-TOPO cloning system (Invitrogen). The pooled plasmid library with $>10,000$ genomic DNA clones was used to transform E. coli BW117N by electroporation. Transformants that were resistant to the dominant

Table 4 E. coli strains and plasmids used in this study

\begin{tabular}{|c|c|c|}
\hline & Relevant genotype & Source or construction \\
\hline \multicolumn{3}{|l|}{ E. coli strains } \\
\hline \multirow[t]{2}{*}{ BW27784 } & $\Delta($ araBAD $) 567 \triangle(r h a B A D) 568$ & Yale E. coli Genetic Stock Center \\
\hline & $\Delta(\operatorname{araFGH}) \Phi\left(\triangle \operatorname{araEpP} \mathrm{CP} 18_{1}-\operatorname{araE}\right)$ & [32] \\
\hline BW117N & BW27784 with chromosomally integrated YpTOP1-D117N gene & {$[10]$} \\
\hline AQ4335 & Dara leu7697 NBRP & NBRP-E. coli at NIG \\
\hline FB20344 & MG1655 ydeA::Tn5KAN-I-Scel & U. Wisconsin [34] \\
\hline YT103 & AQ4335 ydeA::Tn5KAN-I-Scel & P1(FB20344) × AQ4335, Kan ${ }^{r}$ \\
\hline JW1328-1 & $\Delta f n r 771:: k a n$ & Yale E. coli Genetic Stock Center [35] \\
\hline JW1650-1 & $\triangle p u r R 746:: k a n$ & Yale E. coli Genetic Stock Center [35] \\
\hline$\overline{\text { IFL6 }}$ & BW27784 $\triangle$ fnr771::kan & P1(JW1328-1) × BW27784, Kan'r \\
\hline IFL7 & BW27784 $\triangle$ purR746::kan & P1(JW1650-1) × BW27784, Kan' \\
\hline \multicolumn{3}{|l|}{ Plasmids } \\
\hline pAYTOP128 & Mutant derivative of pAYTOP encoding YpTOP1 with G122S, M326V and A383P mutations & [11] \\
\hline$\overline{\mathrm{pCRII}}$ & High copy number cloning vector & Invitrogen \\
\hline pAQ5 & pCR-XL-TOPO cloning product of E. coli chromosome fragment 2618398-2620765 & This study \\
\hline pAQ5-1 & $\mathrm{pCR}-\mathrm{XL}-\mathrm{TOPO}$ carrying upp gene and the intergenic region of upp-purMN & This study \\
\hline pAQ5-2 & pCR-XL-TOPO carrying purM gene and the intergenic region of upp-purMN & This study \\
\hline plnter & $\mathrm{pCR}-\mathrm{XL}-\mathrm{TOPO}$ carrying the intergenic region of upp-purMN & This study \\
\hline plnterD1 & plnter with the FNR binding site deleted & This study \\
\hline plnterD2 & plnter with the PurR binding site deleted & This study \\
\hline
\end{tabular}


lethal effect of YpTOP1-D117N were selected by plating on LB plates with antibiotics and $0.002 \%$ arabinose. Plasmid was isolated from viable colonies and confirmed in subsequent transformation of BW117N to confer resistance to cell killing mediated by topoisomerase I cleavage complex accumulation.

\section{Cell viability assays}

Transformants of BW27784 or BW117N were grown in LB medium with antibiotics to exponential phase $\left(\mathrm{OD}_{600}=0.4\right)$. The cultures were treated with either arabinose to induce recombinant mutant topoisomerase I or the gyrase inhibitor norfloxacin for the stated length of time at $37^{\circ} \mathrm{C}$ with shaking at $215 \mathrm{rpm}$ unless otherwise stated. Serial dilutions of the cultures were then plated on LB plates with antibiotics with $2 \%$ glucose added for BW117N or BW27784 transformed with pAYTOP128, and incubated overnight. The viable colony counts from the treated cultures were normalized against the untreated culture to calculate the survival ratio. The results shown represent the average and standard errors of at least three experiments.

\section{Western blot analysis of recombinant $Y$. pestis topoisomerase I expression}

Exponential phase cultures were treated with indicated concentration of arabinose for 2 or $2.5 \mathrm{~h}$. Cells were collected by centrifugation from volumes based on $\mathrm{OD}_{600}$ and resuspended in SDS gel sample buffer before boiling for $5 \mathrm{~min}$ and SDS page for total protein analysis. The coomassie blue stained gel was examined to confirm equal loading. For improved control of equal loading in experiments using minimal media, total soluble proteins were prepared and quantitated by the BioRad Dc protein assay. Mouse monoclonal antibodies against E. coli topoisomerase I were used in Western blot analysis to detect the highly homologous $Y$. pestis topoisomerase I. Partially degraded $Y$. pestis topoisomerase I (YpTOP*) was also detected.

\section{Hydroxyl radicals formation assay}

BW27784 transformed with vector or pInter was grown to exponential phase in LB before treatment with 250 $\mathrm{ng} / \mathrm{ml}$ norfloxacin, or left untreated as control. After the indicated time, hydroxyl radicals were measured with the fluorescent reporter dye, 3'(p-hydroxyphenyl) fluorescence (HPF) in a FACScan flow cytometer (Becton Dickinson) [13].

\section{Conclusions}

We demonstrated that titration of the E. coli transcription factors FNR and PurR by plasmid clones with the transcription factor binding sites can confer resistance to cell killing mediated by mutant topoisomerase I cleavage complex and norfloxacin acting on DNA gyrase. Our study showed that perturbation of the global regulator FNR and PurR function as well as increase in purine nucleotide availability, could affect the oxidative damage cell death pathway initiated by topoisomerase cleavage complex. The metabolic state of the cell is likely to be an important factor for the bactericidal outcome in this cell death pathway.

\section{Acknowledgements}

We acknowledge NBRP-E. coli at NIG and the Yale E. coli Genetic Stock Center for providing strains. This study was funded by NIH grant R01Al069313 to Yuk-Ching Tse-Dinh.

\section{Authors' contributions}

IL identified and characterized the relevant plasmid clones and E. coli mutants and participated in experimental design, data analysis and manuscript drafting. SA participated in the flow cytometry experiment, data analysis and manuscript drafting. YT conceived of the study, participated in experimental design, data analysis and manuscript drafting. Additionally, all authors have read and approved the final manuscript.

Received: 29 September 2011 Accepted: 12 December 2011 Published: 12 December 2011

\section{References}

1. Schoeffler AJ, Berger JM: DNA topoisomerases: harnessing and constraining energy to govern chromosome topology. Q Rev Biophys 2008, 41(1):41-101.

2. Liu LF: DNA topoisomerase poisons as antitumor drugs. Annu Rev Biochem 1989, 58:351-375.

3. Bernard $\mathrm{P}$, Couturier M: Cell killing by the F plasmid $\mathrm{CcdB}$ protein involves poisoning of DNA-topoisomerase II complexes. J Mol Biol 1992, 226(3):735-745.

4. Hooper DC: Quinolone mode of action. Drugs 1995, 49(Suppl 2):10-15.

5. Drlica K: Mechanism of fluoroquinolone action. Curr Opin Microbiol 1999, 2(5):504-508.

6. Goswami M, Mangoli SH, Jawali N: Involvement of reactive oxygen species in the action of ciprofloxacin against Escherichia coli. Antimicrob Agents Chemother 2006, 50(3):949-954.

7. Dwyer DJ, Kohanski MA, Hayete B, Collins JJ: Gyrase inhibitors induce an oxidative damage cellular death pathway in Escherichia coli. Mol Syst Biol 2007, 3:91.

8. Kohanski MA, Dwyer DJ, Hayete B, Lawrence CA, Collins JJ: A common mechanism of cellular death induced by bactericidal antibiotics. Cell 2007, 130(5):797-810

9. Wang $X$, Zhao $X$ : Contribution of oxidative damage to antimicrobial lethality. Antimicrob Agents Chemother 2009, 53(4):1395-1402.

10. Cheng B, Annamalai T, Sorokin E, Abrenica M, Aedo S, Tse-Dinh YC: Asp-toAsn substitution at the first position of the DxD TOPRIM motif of recombinant bacterial topoisomerase I is extremely lethal to $E$. coli. $J \mathrm{Mol}$ Biol 2009, 385(2):558-567.

11. Cheng B, Shukla S, Vasunilashorn S, Mukhopadhyay S, Tse-Dinh YC: Bacterial cell killing mediated by topoisomerase I DNA cleavage activity. J Biol Chem 2005, 280(46):38489-38495.

12. Sutherland JH, Cheng B, Liu IF, Tse-Dinh YC: SOS induction by stabilized topoisomerase IA cleavage complex occurs via the RecBCD pathway. J Bacteriol 2008, 190(9):3399-3403.

13. Liu IF, Annamalai T, Sutherland JH, Tse-Dinh YC: Hydroxyl radicals are involved in cell killing by the bacterial topoisomerase I cleavage complex. J Bacterio/ 2009, 191(16):5315-5319.

14. Partridge JD, Scott C, Tang Y, Poole RK, Green J: Escherichia coli Transcriptome Dynamics during the Transition from Anaerobic to Aerobic Conditions. J Biol Chem 2006, 281(38):11230-11237.

15. Partridge JD, Sanguinetti G, Dibden DP, Roberts RE, Poole RK, Green J: Transition of Escherichia coli from Aerobic to Micro-aerobic Conditions 
Involves Fast and Slow Reacting Regulatory Components. I Biol Chem 2007, 282(15):11230-11237.

16. Kang Y, Weber KD, Qiu Y, Kiley PJ, Blattner FR: Genome-wide expression analysis indicates that FNR of Escherichia coli K-12 regulates a large number of genes of unknown function. J Bacteriol 2005, 187(3):1135-1160.

17. Salmon K, Hung SP, Mekjian K, Baldi P, Hatfield GW, Gunsalus RP: Global gene expression profiling in Escherichia coli K12. The effects of oxygen availability and FNR. J Biol Chem 2003, 278(32):29837-29855.

18. Scott C, Partridge JD, Stephenson JR, Green J: DNA target sequence and FNR-dependent gene expression. FEBS Lett 2003, 541(1-3):97-101.

19. Grainger DC, Aiba H, Hurd D, Browning DF, Busby SJ: Transcription factor distribution in Escherichia coli: studies with FNR protein. Nucleic Acids Res 2007, 35(1):269-278.

20. Eiglmeier K, Honore N, luchi S, Lin EC, Cole ST: Molecular genetic analysis of FNR-dependent promoters. Mol Microbiol 1989, 3(7):869-878.

21. He B, Shiau A, Choi KY, Zalkin H, Smith JM: Genes of the Escherichia coli pur regulon are negatively controlled by a repressor-operator interaction. J Bacteriol 1990, 172(8):4555-4562.

22. Spiro $S$, Guest JR: FNR and its role in oxygen-regulated gene expression in Escherichia coli. FEMS Microbiol Rev 1990, 6(4):399-428.

23. Wilson HR, Turnbough $\mathrm{CL} \mathrm{Jr}$ : Role of the purine repressor in the regulation of pyrimidine gene expression in Escherichia coli K-12. J Bacteriol 1990, 172(6):3208-3213.

24. Cho BK, Federowicz SA, Embree M, Park YS, Kim D, Palsson BO: The PurR regulon in Escherichia coli K-12 MG1655. Nucleic Acids Res 2011.

25. Dwyer DJ, Kohanski MA, Collins JJ: Role of reactive oxygen species in antibiotic action and resistance. Curr Opin Microbiol 2009, 12(5):482-489.

26. Boysen A, Moller-Jensen J, Kallipolitis B, Valentin-Hansen P, Overgaard M: Translational regulation of gene expression by an anaerobically induced small non-coding RNA in Escherichia coli. J Biol Chem 2010, 285(14):10690-10702.

27. Durand S, Storz G: Reprogramming of anaerobic metabolism by the FnrS small RNA. Mol Microbiol 2010, 75(5):1215-1231.

28. Kiley PJ, Beinert H: Oxygen sensing by the global regulator, FNR: the role of the iron-sulfur cluster. FEMS Microbiol Rev 1998, 22(5):341-352.

29. Malik M, Hussain S, Drlica K: Effect of anaerobic growth on quinolone lethality with Escherichia coli. Antimicrob Agents Chemother 2007, 51(1):28-34

30. Kumari S, Beatty CM, Browning DF, Busby SJ, Simel EJ, Hovel-Miner G, Wolfe AJ: Regulation of acetyl coenzyme A synthetase in Escherichia coli. J Bacteriol 2000, 182(15):4173-4179.

31. Lobell RB, Schleif RF: DNA looping and unlooping by $\mathrm{AraC}$ protein. Science 1990, 250(4980):528-532.

32. Khlebnikov A, Datsenko KA, Skaug T, Wanner BL, Keasling JD: Homogeneous expression of the P(BAD) promoter in Escherichia coli by constitutive expression of the low-affinity high-capacity AraE transporter. Microbiology 2001, 147(Pt 12):3241-3247.

33. Aravind L, Leipe DD, Koonin EV: Toprim-a conserved catalytic domain in type IA and II topoisomerases, DnaG-type primases, OLD family nucleases and RecR proteins. Nucleic Acids Res 1998, 26(18):4205-4213.

34. Kang Y, Durfee T, Glasner JD, Qiu Y, Frisch D, Winterberg KM, Blattner FR: Systematic mutagenesis of the Escherichia coli genome. J Bacteriol 2004, 186(15):4921-4930.

35. Baba T, Ara T, Hasegawa M, Takai Y, Okumura Y, Baba M, Datsenko KA, Tomita M, Wanner BL, Mori H: Construction of Escherichia coli K-12 inframe, single-gene knockout mutants: the Keio collection. Mol Syst Biol 2006, 2, 2006.0008.

doi:10.1186/1471-2180-11-261

Cite this article as: Liu et al:: Resistance to topoisomerase cleavage complex induced lethality in Escherichia coli via titration of transcription regulators PurR and FNR. BMC Microbiology 2011 11:261.

\section{Submit your next manuscript to BioMed Central and take full advantage of:}

- Convenient online submission

- Thorough peer review

- No space constraints or color figure charges

- Immediate publication on acceptance

- Inclusion in PubMed, CAS, Scopus and Google Scholar

- Research which is freely available for redistribution

Submit your manuscript at www.biomedcentral.com/submit
Biomed Central 